\title{
Hospital Acquired Infection: Role of Hand Washing
}

\author{
Subhashish Das* \\ Department of Pathology, Sri Devaraj Urs Medical College, India
}

Received: 嘴 August 27, 2018; Published: 制 August 29, 2018

*Corresponding author: Subhashish Das, Department of Pathology, Sri Devaraj Urs Medical College, Tamaka, Kolar, India

Abbreviations: WHO: World Health Organization; HAIs: Healthcare Associated Infections; ICU: Intensive Care Units; HH: Hand hygiene; HCW: Health-Care Workers

\section{Introduction}

The World Health Organization (WHO) has declared October 15 as Global Hand washing Day, with the aim of raising awareness on the importance of this art and to inculcate the tenet of its basic principles. In fostering this principle, the WHO introduced five critical moments of hand hygiene practice. These include washing hands before touching a patient, before performing cleaning procedures, after coming in contact with infectious materials such as body fluid (urine, feces, sputum), after touching a patient, and after touching a patient surroundings. In hospitals often, greater attention is placed on the doctors and nurses however, other supporting staffs such as the ward attendant, clinical assistant, and laboratory attendants play vital role to patient recovery [1]. Ward attendants are involved in hazardous jobs which expose them to the risk of infection; they also handle patients and their items; hence, they may become vehicle for spread of nosocomial infection, so knowledge of the principles of hand hygiene and handwashing should be paramount in this category of health workers. Similarly, laboratory attendants are exposed to infectious biproducts; them to health hazard [2].

Healthcare-Associated Infections (HAIs) have been a problem worldwide affecting a significant number of hospitalized patients. World Health Organization (WHO) had estimated that globally, $7 \%-12 \%$ of hospitalized patients will acquire health HAIs. International Nosocomial Infection Control Consortium in 2007 had reported an overall HAI incidence rate of 4.4\% corresponding to 9.06 infections per 1000 Intensive Care Units (ICU) days from 7 Indian cities [1]. Simplicity is deceptive. Truly, important things are simple. Simple things are difficult to implement, particularly by doctors. Doctors prefer the challenge of complicated things. This is tragically illustrated by the history of handwashing and hospital infections. Evidence on the effectiveness of the simple procedure if handwashing was first collected by Oliver Wendell Holmes and Ignaz Semmelweis. Holmes after publishing a paper in 1843 on the effectiveness of handwashing in an obscure medical journal devoted himself to poetry which brought him more fame than medicine. Subsequently pioneering work on the efficacy of handwashing for preventing puerperal fever was done by Semmelweis [3].

Patients in the ICUs are more likely to be colonized or infected during their hospital stay. Most of these infections are transmitted to the patients by carriage of microorganisms on the hands of Health-Care Workers (HCW) while handling patients. Hand hygiene $(\mathrm{HH})$ is one of the most important measures to prevent HAIs. HH is a general term that applies to handwashing, antiseptic hand wash, alcohol-based hand rub, or surgical $\mathrm{HH}$ /antisepsis. While these measures as simple and inexpensive as handwashing with soap and water can be implemented to reduce HAIs and save millions of lives, compliance with it is still low is developing countries. This may be attributed to lack of proper knowledge and recognition of HH opportunities during patient care [4].

To address this problem, continuous efforts are being made to identify effective and sustainable strategies. One of such efforts, is the introduction of an evidence-based concept of "My five moments for HH" by World Health Organization which includes use of $\mathrm{HH}$ before touching a patient, before performing aseptic and clean procedures, after being at risk of exposure to body fluids, after touching a patient, and after touching patient surroundings [4]. In India, technology to monitor adherence may not be available at every hospital setting; direct observation remains the gold standard. With this aim, we attempted to assess HH compliance 
among HCW in the ICU of a tertiary care super specialty institute in North India by direct observation method [5]. Hospital-acquired infection (HAI) or health care-associated infection is an infection occurring in a patient during the process of care in a hospital or other health-care facility which was not present incubating at the time of admission. Hand hygiene ( $\mathrm{HH})$ is a simple yet effective way to prevent HAIs. Studies have shown that there is knowledge about the importance of $\mathrm{HH}$, but implementation of these guidelines in practice is poor.

This is evident from the high incidence of HAIs both in developing and developing countries. According to the centers for Disease Control and Prevention (CDC) data, approximately one in 25 patients acquire HAI during their hospital care in the USA, adding up to about 7,22,000 infections a year, out of which, 75,000 patients die of their infections. In developed countries, HAIs, continue to account for complications in 5\%-10\% of admissions to acute-care hospitals. In developing countries, the impact of HAI is far greater, approximately two-to twenty-fold higher than those in the developing countries. Although the surveillance is poor in developing countries, the prevalence studies report hospital-wide infection rates usually higher than $15 \%[6,7]$. The gold standard of monitoring $\mathrm{HH}$ compliance is direct observation, but the reliability of this method to measure true, actual compliance with $\mathrm{HH}$ is questionable. HH compliance increases in front of the observes. It is the classic Hawthorne effect, where people change their behavior when they know they are being watched. Another issue is that it is difficult to observe all the HH opportunities that a health-care provider should undergo. Health-care providers might need to clean their hands as many as 100 times per 12-h shift, depending on the number of patients and intensity of care. However, studies show that on an average, health-care providers clean their less than half of the times they should.

Another novel intervention for monitoring $\mathrm{HH}$ that has been tired in Brazil involve using electronic surveillance system with radiofrequency devices with censors placed at patient bedside, hand rub dispenser, and employee's ID badge. Such newer interventions for better monitoring and encouraging $\mathrm{HH}$ among HCWs can go a long way in decreasing HAL's prevalence in the health-care settings [8]. The Centers for Disease Control and Prevention and World Health Organization both maintain extensive guidelines to inform healthcare providers about the proper practices of hand hygiene. Additionally, various quality and patient safety organization such as the Institute for Healthcare improvement and the joint Commission have published ancillary resources to assist facilities with implementation of the latest evidence-based practices.

A solid hand hygiene program with documented compliance with reduce risk for transmission of microorganisms to patient, reduce risk for healthcare worker colonization, and reduce the mortality, morbidity and costs associated with treatment of healthcare-acquired infections [3]. The goal of hand washing and hand hygiene is to remove as many microorganisms from the hand as possible to avoid transmission. The skin flora is divided into layers: transient and resident. The transient flora colonizes the most superficial layers of the skin (epidermis) and is fairly easily removed from the hand after thorough hand hygiene is performed [3]. According to the latest guidelines from the WHO, healthcare workers should practice proper hand hygiene before touching he patient, after touching inanimate objects in the patient's surroundings, after exposure to bodily fluids and before clean/ aseptic procedures.

\section{Transmission of microorganisms via the hands of healthcare workers}

The 2009 World Health Guidelines for Hand Hygiene for Healthcare settings identifies five ways [9] the nosocomial transmission of pathogens from one patient to another via a healthcare worker's hands can occur.

a) Microorganism must be present on the patient's skin or have to be shed into the patient's environment such as nearby inanimate objects, such as the bedside table.

b) Microorganisms must be transferred to the hands of the healthcare worker

c) The microorganisms that have contaminated the worker's hands must be able to survive on the hands.

d) The healthcare worker must either omit hand hygiene or inadequately perform it.

e) The healthcare worker's contaminated hands must come into direct contact with either another patient or with a inanimate object that will come into contact with a patient, such as a shared portable vital signs monitor.

\section{Observed risk factors for poor adherence to recommended hand-hygiene practices include}

a) Physician status

b) Male sex

c) Nursing assistant

d) Working in an intensive care setting

e) Wearing gowns/gloves

f) Automated sinks

g) Activities associated with high risk of cross-contamination [8]

\section{Chemical agents for hand washing}

a) Surgical hand scrubs- an antiseptic containing preparation that substantially reduces the number of microorganisms on intact skin [4]. 
b) Antiseptic hand washers- antiseptics containing preparation designed for frequent use, reducing the number of microorganisms on intact skin to an initial baseline level after adequate washing, rinsing, and drying.

c) Patient preoperative skin preparations-fast acting, broadspectrum and persistent antiseptics containing preparation that reduce the number of microorganisms.

\section{Alcohol based agents may be used in the following cases}

a) When hands are not visibly soiled or contaminated with blood or body fluids [9].

b) Before direct contact with patients.

c) After direct contact with a patient's skin.

d) After contact with bodily fluids or excretions, mucous membranes, broken skin or would dressings.

e) When moving from a contaminated to a clean body site.

f) After contact with an inanimate environment (equipment or furniture near patient). g) After removing gloves.

h) Emergency situations where sinks are not available (EMS, Police, Fire Rescue) and hands are visibly soiled.

\section{Selection of hand hygiene agents}

Hand hygiene agents must be carefully selected in order to ensure compliance, efficacy and safety for both the healthcare workers and patient. Several factors must be taken into consideration [8]:

a) Cost of the agent

b) Ease of use for healthcare workers

c) Potential for dermal irritation and skin reactions

d) Efficacy of the agent

e) Product availability and accessibility within the healthcare facility

f) Drying time necessary after applying the agent

g) Staff support for the selected product

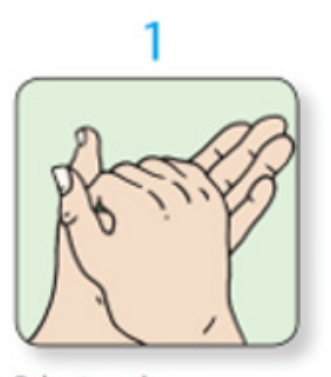

Palm to palm.

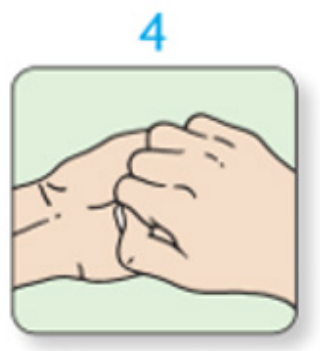

Backs of fingers to opposing palms with fingers interlocked.

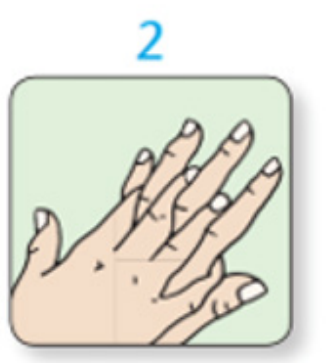

Right palm over left dorsum and left palm over right dorsum.

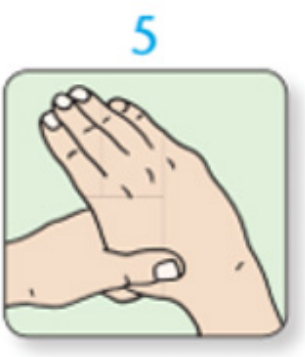

Rotational rubbing of right thumb clasped in left palm, then vice versa.

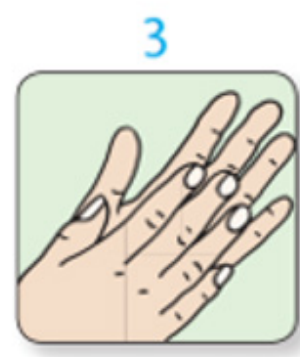

Palm to palm, fingers interlaced.

6

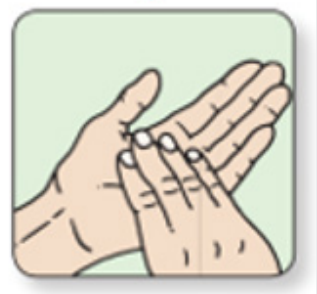

Rotational rubbing. backwards and

forwards with clasped

fingers of hand in left

palm then vice versa.

Figure 1: Showing the Essential Steps of Hand Washing.

All too often, the hands of the patients and/or residents are neglected while in a healthcare facility. The contaminated hands of the patient can serve as not only a source for transmission, but also as a reservoir for microbial growth. The acuity of many of today's hospitalized patients is extremely high, with some completely non- ambulatory and confined to bed. Hand hygiene dispensers located on a wall away from the patient are not ideal for situations where the patient may not be able to walk to the dispenser. It is critical to place hand hygiene solutions at the point-of-care and use, which in many cases is directly at the patient's bedside. This will improve 
compliance for patient hand hygiene, and more importantly serve as a constant reminder of the importance of hand hygiene for all that are involved with the care of the patient. Healthcare providers should encourage not only the patient, butalso their family members and visitors, to regularly practice hand hygiene frequently when indicated to minimize contamination and potential [10]. Monitoring of hand hygiene is a key component in improving processes and compliance. The use of "secret shoppers" has proven to be an effective measurement tool for some facilities, while others rely on different measures to gauge compliance. Monitoring should be conducted on a routine bases and documented. Any personnel not compliant with the hand hygiene standards should be immediately, counseled to ensure prompt remediation (Figures 1 \& 2). The IHI has created an entire resource guide centered on the proper methods of monitoring hand hygiene and judging compliance [11].

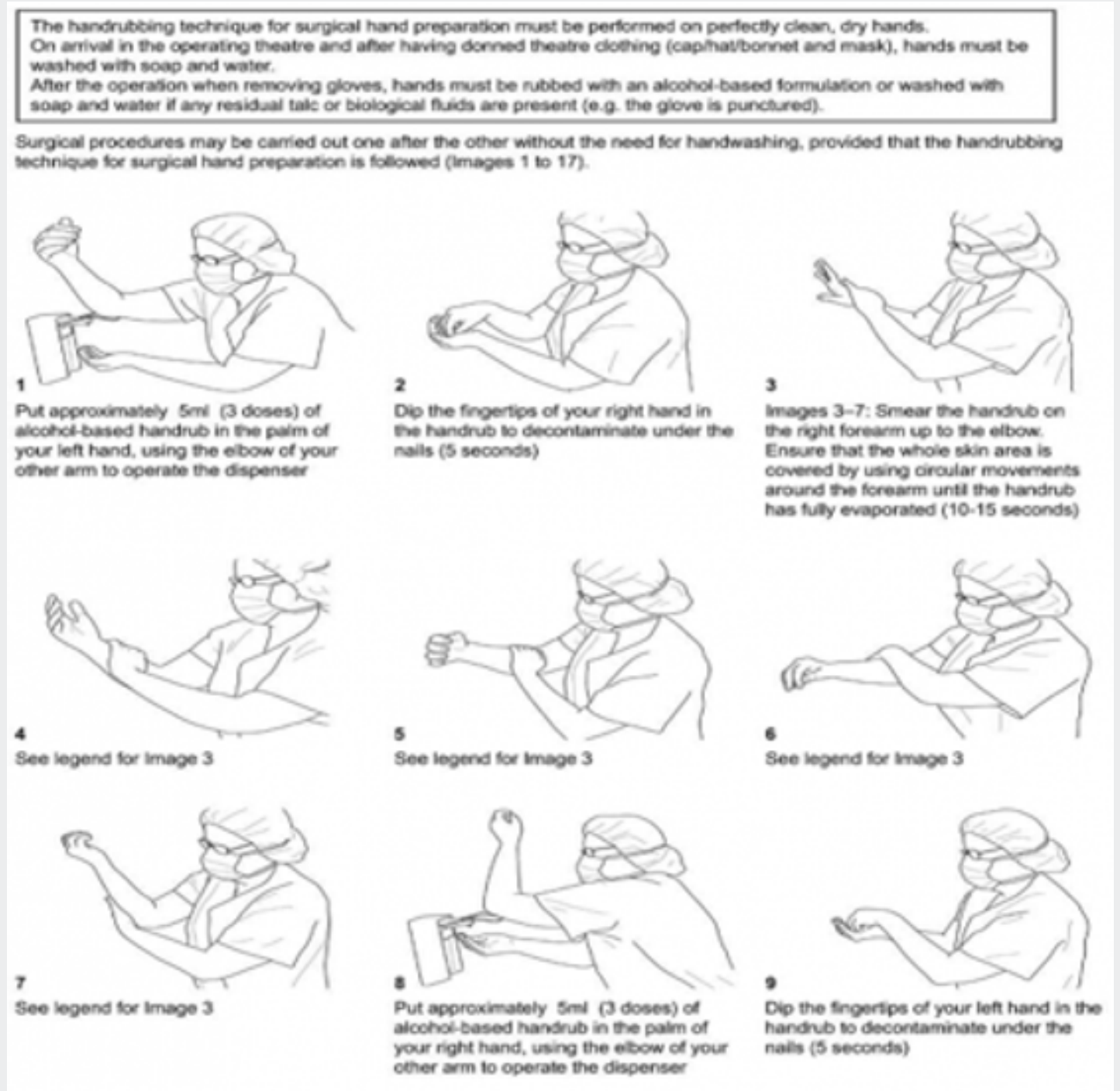

Figure 2: Showing the Essential Steps of Hand Washing.

\section{References}

1. Benenson AS (1995) Control of communicable diseases manual. American Public Health Association Washington.

2. Tikhomirov E (1987) WHO Programme for the Control of Hospital Infections. Chemiotherapia 3: 148-151.

3. Lane HJ, Blum N, Fee E (2010) Oliver Wendell Holmes and Ignaz Philipp Semmelweis: Preventing the transmission of puerperal fever. Am J Public Health 100: 1008-1009.

4. Mayon White RT (1988) An international survey of the prevalence of hospital-acquired infection. J Hosp Infect 11: 43-48.
5. Ponce de Leon S (1991) The needs of developing countries and the resources required. J Hosp Infect 18: 376-381.

6. Pittet D, Allegranzi B, Sax H (2006) Evidence based model for hand transmission during patient care and the role of improved practices. Lancet Infect Dis 6(10): 641-652.

7. Kretzer EK, Larson EL (1998) Behavioral interventions to improve infection control practices. Am J Infect Control 26(3): 245-253.

8. Whitby M, McLaws M L, Ross RW (2006) Why healthcare workers don't wash their hands: A behavioral explanation. Infect Control Hosp Epidemiol 27(5): 484-492. 
9. Rupp ME, Fitzgerald T, Puumala S (2008) Prospective, controlled, crossover trial of alcohol-based hand gel in critical care units. Infect Control Hosp Epidemiol 29(1): 8-15.

10. Picheansathian W (2004) A systematic review on the effectiveness of alcohol-based solutions for hand hygiene. Int J Nurs Pract 10(1): 3-9.
11. Conly JM, Hill S, Ross J (1989) Handwashing practices in an intensive care unit: The effects of an educational program and its relationship to infection rates. Am J Infect Control 17(6): 330-339.
(C) (i) This work is licensed under Creative

To Submit Your Article Click Here:

DOI: $10.32474 /$ SCSOAJ.2018.01.000117

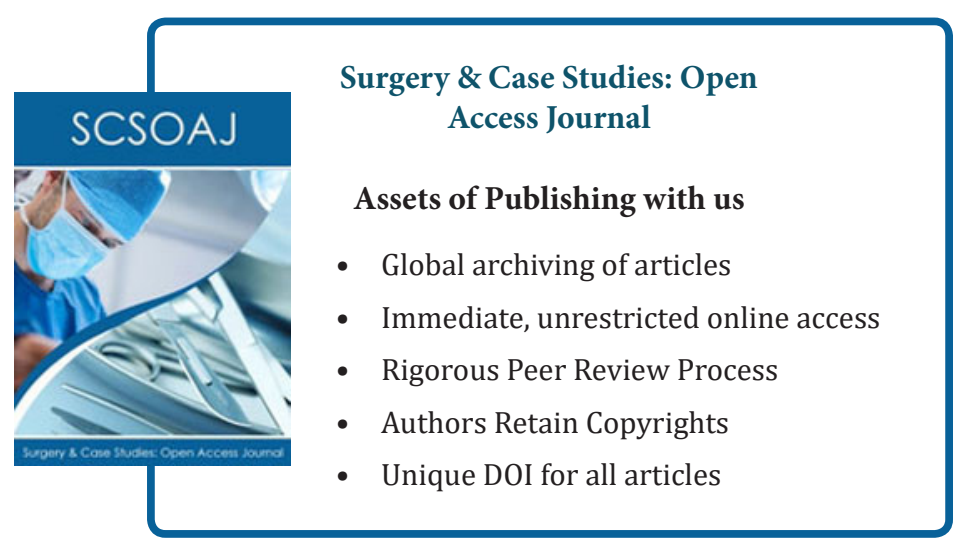

